\title{
Signaling Properties of Ascaris Motorneurons: Graded Active Responses, Graded Synaptic Transmission, and Tonic Transmitter Release
}

\author{
Ralph E. Davis' and Antony O. W. Stretton ${ }^{1,2}$ \\ 'Neurosciences Training Program, and 'Department of Zoology, University of Wisconsin-Madison, Madison, Wisconsin \\ 53706
}

The commissural motorneurons of the nematode Ascaris are capable of transmitting signals passively over long distances with little decrement. This ability is due to the high resistivities of their membranes (Davis and Stretton, 1989). Although these cells rely on their passive properties for longdistance signaling, voltage-sensitive channels are present in commissural membranes. These channels underlie the graded active responses that can be elicited at the offset of abrupt hyperpolarizing and depolarizing intracellular current pulses. The inhibitory motorneurons generate membrane potential oscillations when they are strongly depolarized. Allor-none action potentials have never been observed to occur spontaneously, nor has it been possible to evoke them even when the cells have been strongly hyperpolarized to remove any possible channel inactivation. Our findings indicate that the typical all-or-none action potentials so commonly used in nerve cells throughout the animal kingdom do not occur in these cells. Synaptic transmission is therefore mediated without spikes and is graded. The resting potentials of Ascaris motorneurons lie where the synaptic input-output curves are steepest, above the threshold for release of neurotransmitter. Tonic transmitter release from commissural motorneurons may be the neural mechanism underlying the hydrostatic skeleton of Ascaris.

The electrical signaling capabilities of a cell are determined by the passive properties of its membrane combined with its repertoire of active (voltage-sensitive) responses. How the cell transfers signals to postsynaptic cells in a neural circuit will be determined by the properties of its synapses. The commissural motorneurons of the Ascaris motornervous system have been used to examine some of these properties.

With regard to the signaling ability of commissural motorneurons, we have determined their cable properties (Davis and Stretton, 1989). The high resistivity of the commissural membrane accounts for the large space constant of these cells and thus their ability to conduct signals over centimeter distances.

\footnotetext{
Received Nov. 9, 1987; revised July 18, 1988; accepted July 22, 1988.

We thank Drs. James Angstadt, Ching Kung, and Donata Oertel for critically reading the manuscript. We are also grateful to Dr. Carl Johnson and Judith Donmoyer for helpful discussions. The manuscript was capably typed by Mrs. Beth Davis. This work was supported by USPHS Grant 15429 .

Correspondence should be addressed to Dr. Antony O. W. Stretton, Department of Zoology, Zoology Research Building, University of Wisconsin-Madison, Madison, WI 53706.

Copyright (C) 1989 Society for Neuroscience $0270-6474 / 89 / 020415-11 \$ 02.00 / 0$
}

In this paper we continue the analysis of commissural motorneurons by describing (1) the active responses that they can generate and (2) the nature of synaptic transmission between commissural motorneurons and their postsynaptic partners.

We conclude that commissural motorneurons can sustain graded, active signals; these include offset responses following strong depolarizing and hyperpolarizing current pulses and rhythmic oscillatory potentials. These neurons do not, however, generate all-or-none spikes under the experimental conditions we have tested. Synaplic transmission is graded and, at normal resting potentials, the synapses are tonically active.

\section{Materials and Methods}

Electrophysiological techniques, the perfusion apparatus, physiological saline, and the nature of the dissection have been described in the previous paper (Davis and Stretton, 1989). All motorneuron recordings were made in the commissure. Veratridine was obtained from the Aldrich Chemical Company (Milwaukee, WI).

\section{Results}

Intracellularly recorded commissural signals: anode- and cathode-break responses

Active responses occurring at the offset of intracellularly injected current pulses indicate that voltage-sensitive channels are present in commissural motorneurons. Prominent among these is the anode-break response that occurs at the offset of intracellular hyperpolarizing current pulses (Fig. 1). These responses have been observed in all 5 commissural motorneuron classes (DE1, DE2, DE3, DI, and VI). Ascaris motorneurons yield graded anode-break responses, as is evident when successive hyperpolarizing pulses of varied amplitude are administered (Fig. 1).

To examine the voltage-sensitive range of the response, an anode-break eliciting pulse was given before, during, and after a longer duration hyperpolarizing pulse (Fig. 2). Although the shorter pulse elicited anode-break responses before and after the longer pulse, it did not do so during the longer pulse. This result suggests that the channels responsible for the anode-break have a voltage-sensitive range above (i.e., more depolarized than) the levels to which these cells had been hyperpolarized during the longer pulse. The lower limit for the activation of these channels is approximately $3-15 \mathrm{mV}$ more negative than the resting potential. Based on the maximal anode-break responses obtained, the voltage-sensitive range might extend to approximately $30 \mathrm{mV}$ more positive than the resting potential. Thus, the voltage-sensitive range of the rising phase conductance(s) begins below and overlaps the resting potential of the cell. Without voltage-clamp analysis it is difficult to characterize 
Figure 1. Anode-break responses obtained at the end of intracellular injection of strong hyperpolarizing currents. Decreasing amplitude of intracellularly injected hyperpolarizing pulses ( $I$, current monitor) results in a graded decrease in the amplitude of the evoked anode-break response (arrowheads) in a DE 1 motorneuron (lower trace).

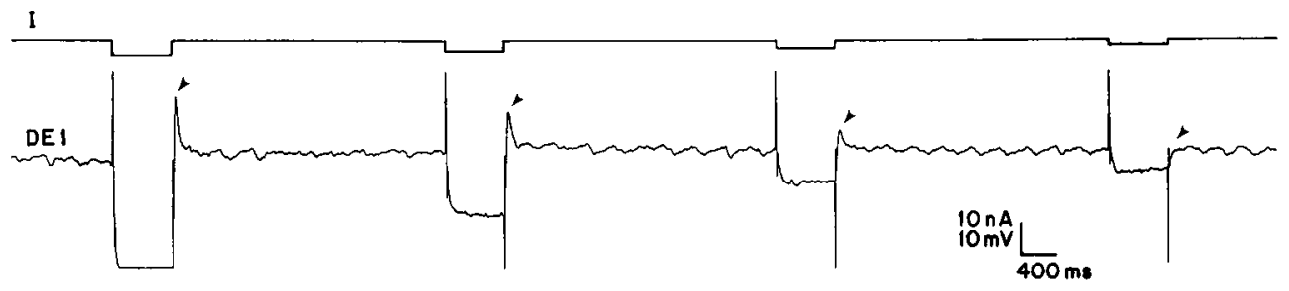

the voltage-sensitive range precisely because of the possibility that more than one conductance contributes to the overall voltage response. Furthermore, the location of voltage-sensitive channels on distant regions of the cell confounds the precise determination of threshold.

In saline in which $\mathrm{Co}^{2+}$ ions were substituted for $\mathrm{Ca}^{2+}$ ions, anode-break responses were reversibly inhibited (Fig. 3). Thus, these responses appear to be $\mathrm{Ca}^{2+}$ dependent. Perhaps the voltage-sensitive channels being activated in such responses are the same as those responsible for the $\mathrm{Ca}^{2+}$ influx necessary for neurotransmitter release and hence are associated with the presynaptic terminals of the neuron. If this were the case, the channels might be entirely localized to the axonal output region of the cell, where synapses to muscle and to other neurons occur (Stretton et al., 1978); conceivably, the commissure itself could be devoid of such channels. In order to test this possibility, the commissure was severed near the dorsal nerve cord with an electrolytically sharpened tungsten needle, thus isolating the commissure from the synaptic output zone located in the dorsal nerve cord. It is still possible to record normal resting potentials in the commissure after cutting, so we presume that its membrane reseals. In these cases, anode-break responses similar to those recorded previously in the uncut commissure were still obtained (Fig. 4). Both before and after the cut, the anode-break response retains its graded nature. This result shows that the voltage-sensitive channels of the cell are present at sites other than its output synapses.

We also observed a cathode-break response that is a hyperpolarization that occurs at the offset of depolarizing pulses (Fig. 5). It has been observed in DE1, DE2, DI, and VI motorneurons. (DE3 has not yet been thoroughly examined.) In quiet preparations, the amplitude and duration of the cathode-break response increase in graded fashion with increasing amplitude or duration of the depolarizing current pulse (Fig. 5).

\section{Intracellularly recorded commissural signals: oscillatory} activity

The most striking examples of voltage-sensitive responses involve the rhythmic, oscillatory signals that have been observed in the DI and VI motorneurons (Fig. 6). These responses have a periodicity ranging from approximately 0.2 to $2 \mathrm{sec}$. From cell to cell the oscillatory signal can vary in shape between a symmetrical waveform resembling a sinusoidal wave and a ramp potential with a superimposed depolarizing response. This variation in the waveform can also occur during the course of recording from a single neuron. Spontaneous oscillatory activity is seen most commonly in Ascaris saline containing $5.9 \mathrm{~mm}$ $\mathrm{Ca}^{2+}, 4.9 \mathrm{~mm} \mathrm{Mg}{ }^{2+}$. In saline containing elevated $\mathrm{Ca}^{2+}$ and $\mathrm{Mg}^{2+}$ levels ( 11.8 and $9.8 \mathrm{mM}$, respectively), VI and DI motorneurons occasionally oscillate but more commonly they are either quiet or show regular, spontaneous IPSPs. In quiet preparations, oscillatory activity can be elicited by depolarizing intracellular pulses of long duration (Fig. 6B).

When an inhibitory motorneuron is oscillating (either spontaneously or as a result of intracellular depolarizing pulses), the muscle cells onto which it makes synapses exhibit membrane potential oscillations that are antiphasic to those occurring in the motorneuron (Fig. 6, B, C). Intracellular hyperpolarizing current pulses reduce the amplitude and frequency of the oscillations, abolishing them at high stimulus strengths (Fig. 6C). When the membrane potential of the neuron is changed, the change in the amplitude of the oscillatory potentials is unlike that which would be expected if excitatory presynaptic inputs were responsible for the signals. For example, injection of depolarizing current pulses would be expected to decrease the amplitude of an EPSP input(s) were it generating the depolarizing phase of a spontaneous oscillation. Conversely, injection of hyperpolarizing current pulses would be expected to enhance the amplitude of such an EPSP input. In contrast, depolarization enhances oscillatory activity and hyperpolarization suppresses this activity. These observations suggest that the oscillations are generated endogenously (see also Angstadt, 1986).

In cells that are exhibiting uniform spontancous oscillations, the phase of the oscillatory rhythm can be reset by injection of current pulses of the appropriate polarity and phasing relative to the rhythm (J. D. Angstadt, R. E. Davis, and A. O. W. Stretton, unpublished observations). While such resetting experiments do not reveal whether the oscillatory rhythm is en-

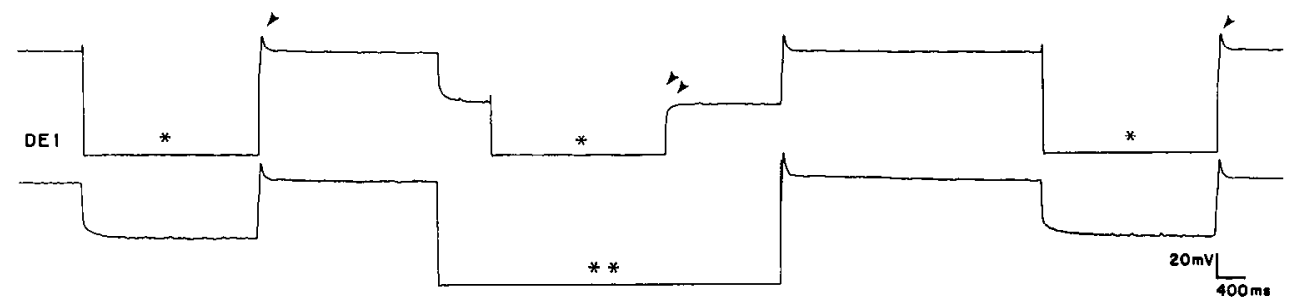

Figure 2. Membrane potential at which anode-break responses can be activated lies near the resting potential. Two microelectrodes were placed in the same DE1 motorneuron. One microelectrode (upper trace, bridge circuitry unbalanced) injected a uniform 2.5 sec hyperpolarizing pulse (*) before, during, and after a $5 \mathrm{sec}$ hyperpolarization $\left(^{* *}\right)$ injected by the second microelectrode (lower trace, bridge circuitry unbalanced). Examination of the upper trace reveals the presence of an anode-break response (single arrowhead) for the $2.5 \mathrm{sec}$ pulses before and after the 5 sec pulse. No anode-break response was elicited during the $5 \mathrm{sec}$ hyperpolarization (double arrowhead). 

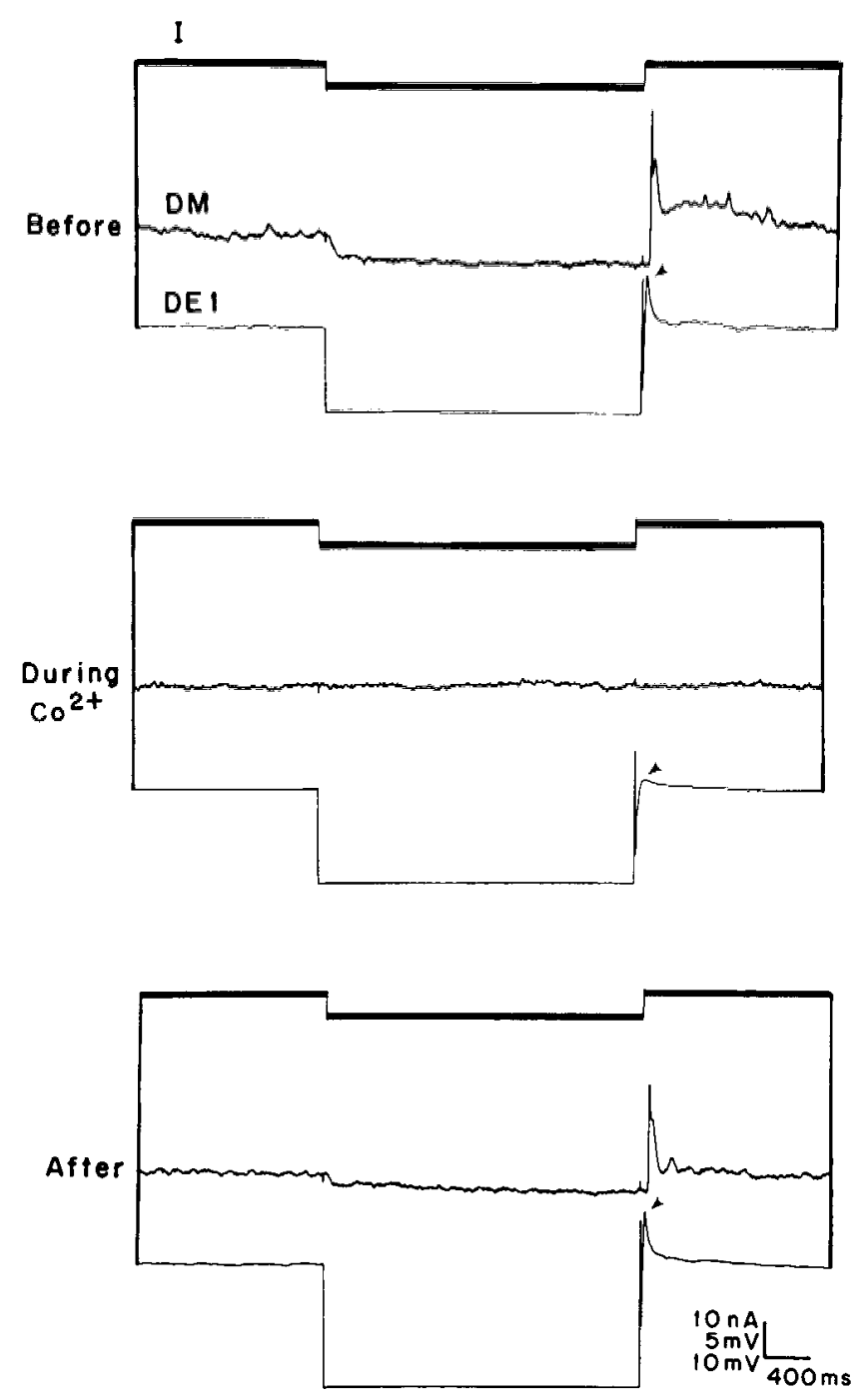

Figure 3. Anode-break response is reversibly blocked by $\mathrm{Co}^{2+}$. A hyperpolarizing current pulse ( $I$, current monitor) capable of eliciting an anode-break response (arrowhead) is injected into a DE1 motorneuron (lower trace, bridge circuitry unbalanced) before, during, and after perfusion with $\mathrm{Co}^{2+}$. The middle trace $(D M)$ is a simultaneous intracellular recording of a muscle cell that receives innervation from the DEl motorneuron. Note that the muscle is hyperpolarized upon the hyperpolarization of $\mathrm{DE} 1$ when bathed in $\mathrm{Ca}^{2+}$ but not $\mathrm{Co}^{2+}$ solution. This result indicates that the muscle is receiving tonic input from DE1. Note also that the muscle response to the anode-break signal in DE1 is reversibly blocked by $\mathrm{Co}^{2+}$ perfusion.

dogenously generated or circuit-generated, they do, however, indicate that these cells are not merely follower cells responding to a rhythm imposed from an outside source.

\section{Absence of all-or-none spikes}

Extracellular suction electrode stimulation of the dendrite of a commissural motorneuron results in an intracellularly recorded motorneuron response that is graded in amplitude with stimulus strength (Fig. 7). The gradedness of responses evoked by extracellular stimulation is characteristic of all the motorneuron classes investigated (DE1, DE2, DI, and VI). Graded responses can be obtained by either direct stimulation of the motorneuron dendrite or by placing the suction electrode at some distance anterior or posterior to the dendrite and stimulating the response

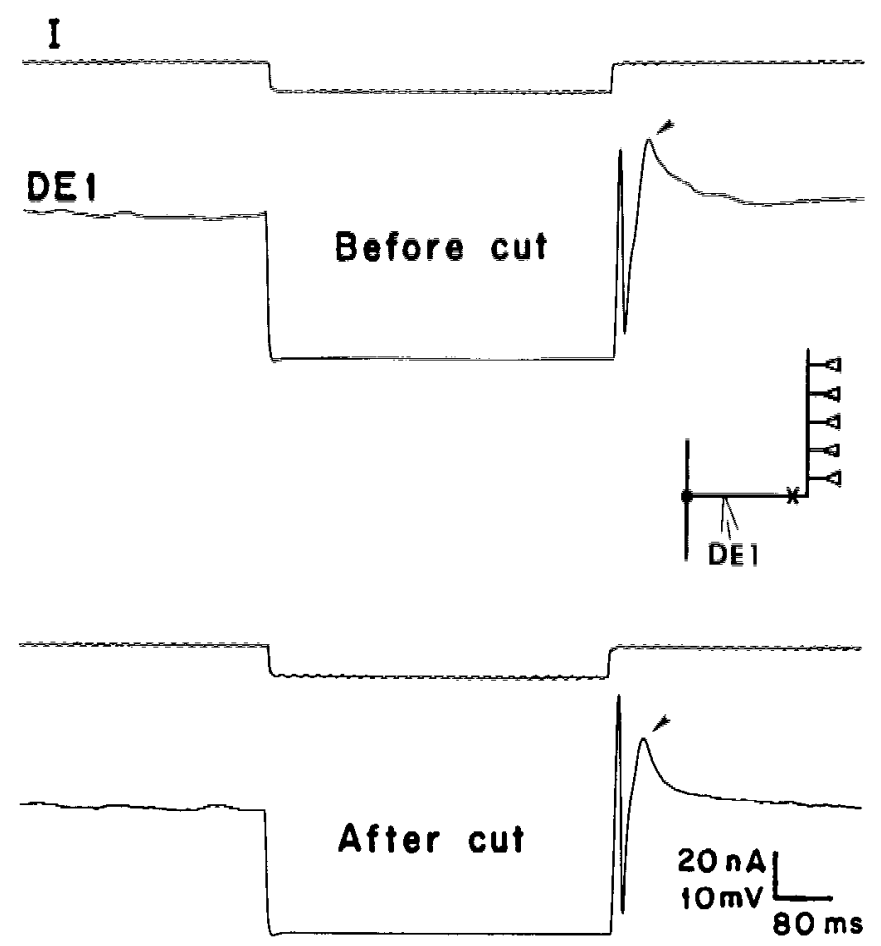

Figure 4. Similar anode-break responses can be elicited in the commissure before and after severing the commissure from its synaptic output zone. A DEl motorneuron (lower trace, bridge circuitry unbalanced) is penetrated near the ventral nerve cord, before and after severing ( $x$ in inset) the commissure from its synaptic output process in the dorsal nerve cord. Anode-break responses (arrowheads) were elicited before and after the cut with identical intracellularly injected current pulses ( $I$, current monitor). The transient prior to the anode-break response is an artifact due to the strong current pulse and, unlike the anode-break response, occurs whether the electrode is in or out of the motorneuron (data not shown).

indirectly by means of ventral nerve cord neuron(s) presynaptic to the motorneurons. In either case, the response amplitude increases in graded fashion with no obvious indication of a threshold. With indirect stimulation, a plateau is reached after which little or no further increase occurs; even though the stimulation voltage continues to be increased (Fig. 7), all-or-none action potentials are not seen. Though the direct response begins to reach a plateau, it does not saturate fully as the indirect response does.

When a current-passing and a recording microelectrode are placed in the same motorneuron, no all-or-none spikes are evoked even when the membrane is depolarized up to $+50 \mathrm{mV}$ above the resting potential; despite the absence of spikes in the motorneuron, strong intracellularly recorded muscle responses are produced by such stimulation (see Figs. 8 and 9 in Davis and Stretton, 1989). Under the experimental conditions used it has not been possible to evoke all-or-none action potentials by extracellular suction electrode stimulation or intracellular injection of strong depolarizing current pulses, nor have they been observed to occur spontaneously in commissural motorneurons. Because of the passive membrane properties of commissural motorneurons (Davis and Stretton, 1989), signals can conduct long distances to activate muscle a centimeter or more from their site of origin. It is possible that all-or-none action potentials are being initiated in the nerve cord axonal processes of these motorneurons, but if so, one would expect them to return to 


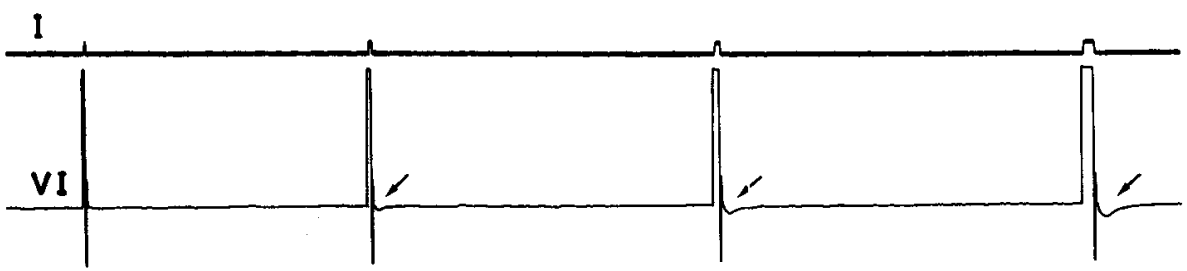

Figure 5. Cathode-break responses obtained after intracellular injection of strong depolarizing currents. Increasing the duration of intracellularly injected depolarizing pulses ( $I$, current monitor) results in graded increases in the amplitude and duration of the cathodebreak response in a VI motorneuron (arrows; lower trace, bridge circuitry unbalanced).

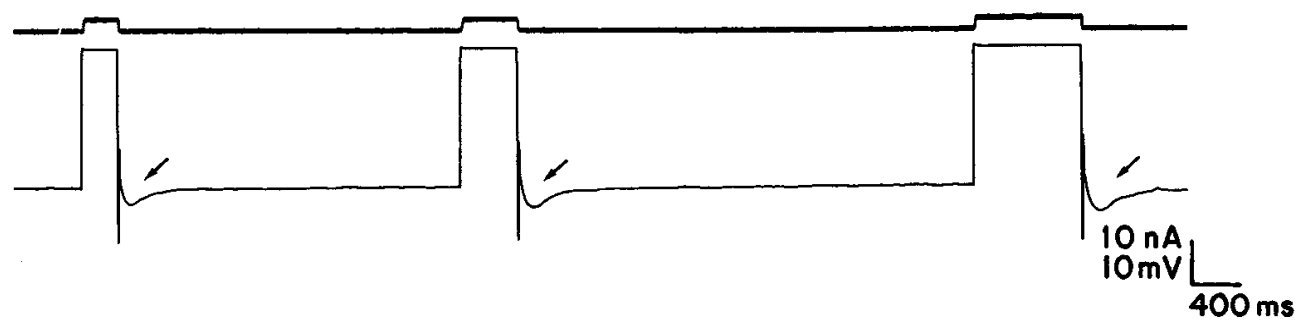

the commissural recording site in some form. The long-distance signaling ability of the commissure and the simple geometry and lack of multiple branch points are features of these cells that should optimize the observation of such signals; however, no such signals have been observed.

Our inability to elicit all-or-none action potentials is not the result of inactivation of the voltage-sensitive channels ( $c f$. Hengstenberg, 1977). We tested this by examining the cells' signals while they were hyperpolarized to approximately $-70 \mathrm{mV}$ (Fig. 8). Maximal suction electrode-evoked responses were evoked before, during, and after such strong hyperpolarizing pulses. There was at most a $3 \mathrm{mV}$ increase in the amplitude of the reponses during the pulses. No all-or-none action potentials were evoked. Similarly, hyperpolarization of a DE2 motorneu- ron does not convert its spontaneous EPSPs into spikes. Some increase in the amplitudes of EPSPs occurs during the pulse, suggesting that EPSPs result from conductance increases whose reversal potentials are near or above $0 \mathrm{mV}$. The signals sum when in phase and reverse during strong depolarizing current pulses.

Veratridine, a blocker of $\mathrm{Na}$ channel inactivation, has been used to demonstrate the presence of a masked spiking mechanism in the normally nonspiking crab stretch receptor (Lowe et al., 1978). When we perfused Ascaris with 50 or $100 \mu \mathrm{M}$ veratridine, there was no indication of a spiking mechanism in Ascaris commissural motorneurons. Resting potentials, suction electrode-evoked responses, anode-break responses, EPSPs, and oscillatory activity all remained unaffected by veratridine.

A

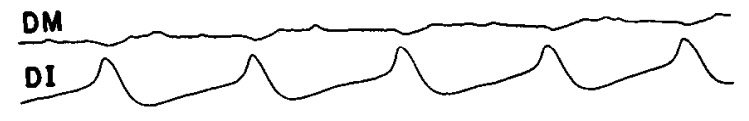

Figure 6. Spontaneous and evoked oscillatory signals observed in inhibitory motorneurons. $A$, Spontaneous ramp potentials (period approx. $1.4 \mathrm{sec}$ ) occurring in a DI motorneuron (lower trace). Dorsal muscle recordings (upper trace) exhibited an IPSP for each DI depolarization. $B$, Oscillatory potentials (period approx. 0.5-1 sec) were induced in this quiet VI cell by injection of a long-duration intracellular depolarizing current pulse (lower trace; approx. $+2 \mathrm{nA}$ of injected current, current trace not shown). Ventral muscle (upper trace) reflected the evoked VI activity with antiphasic oscillatory responses. $C$, Hyperpolarizing current (approx. -2 $n A$, current trace not shown) injected into a spontaneously oscillating VI motorneuron suppresses the oscillations (lower trace). Note the concomitant reduction in ventral muscle oscillatory activity.

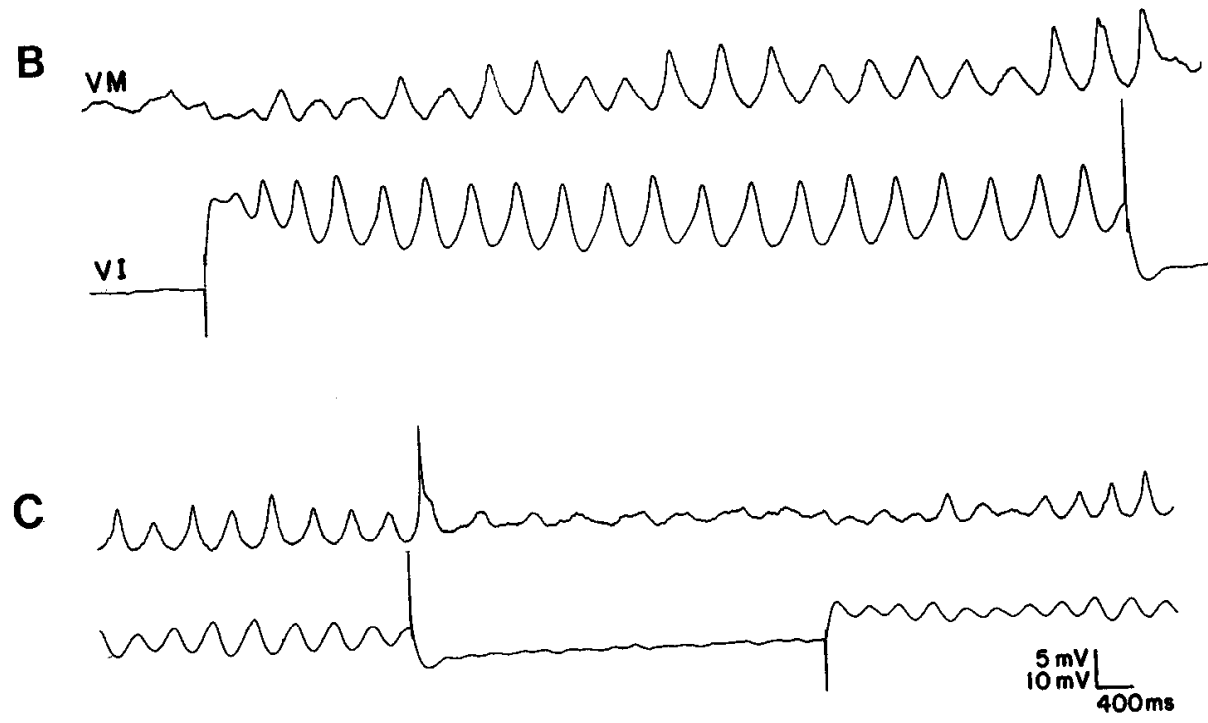




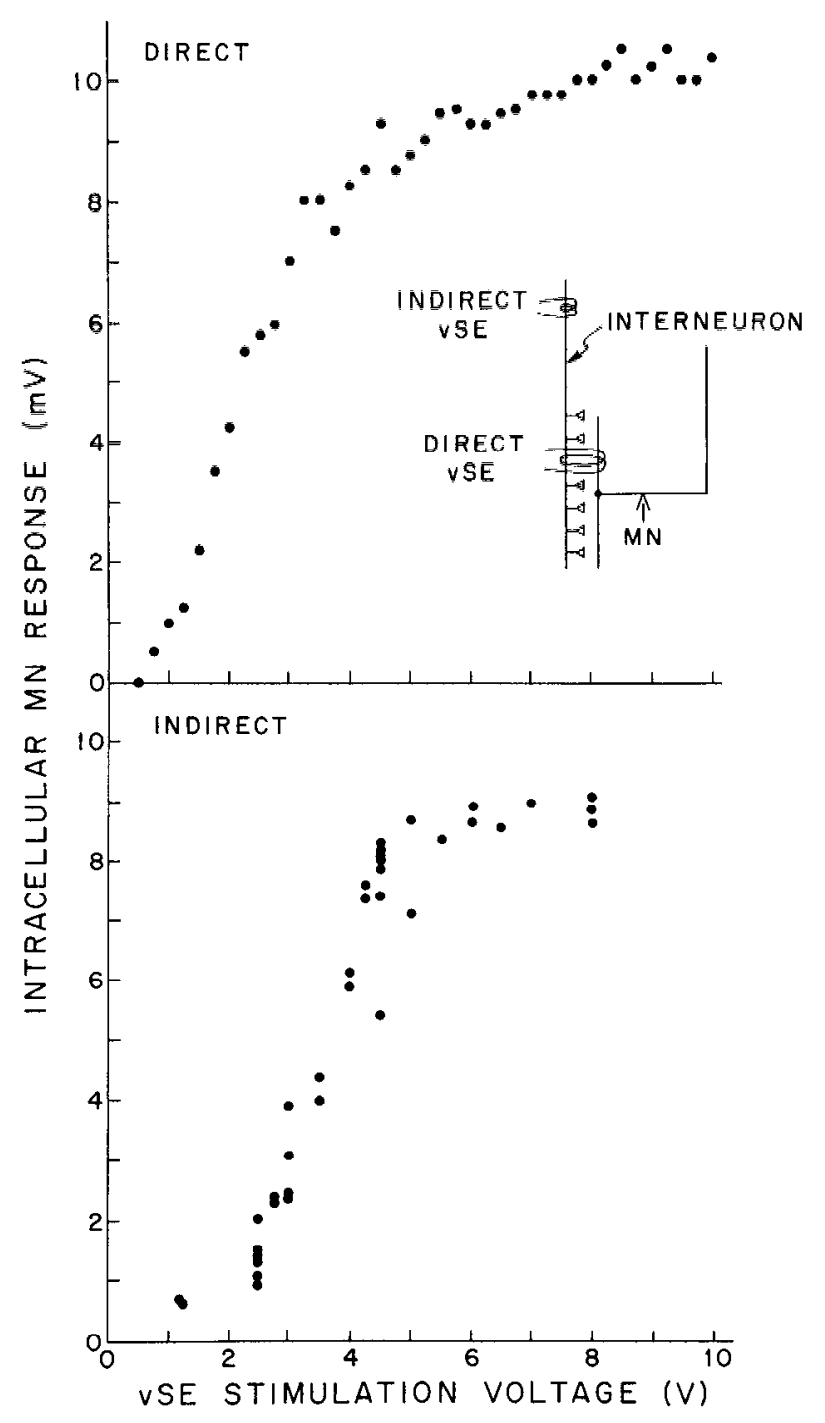

Figure 7. Graphs of ventral suction electrode $(v S E)$ voltage versus motorneuron response amplitude based on direct and indirect responses (see text for additional description).

\section{Graded synaptic transmission}

When depolarized with current, both excitatory and inhibitory motorneurons exhibit graded synaptic transmission at both motorneuron-muscle (Figs. 9, 10) and motorneuron-motorneuron synapses (Figs. 11, 12). Figures 13 and 14 illustrate the inputoutput relationships of representative excitatory and inhibitory motorneuron-muscle synapses. Postsynaptic plateau (steadystate) responses are plotted as a function of depolarizing and hyperpolarizing current injected into the presynaptic motorneuron. The postsynaptic responses are not the result of extracellular current spread since similar or stronger current pulses have no effect on the postsynaptic cell when the stimulating microelectrode is withdrawn just outside the presynaptic motorneuron.

Square depolarizing pulses of current injected into excitatory motorneurons frequently evoke an initial transient depolarization in the postsynaptic cell (Fig. 9). This peak response, like the plateau response following it, is usually graded with depolarizing current. In inhibitory motorneurons, there is no com-

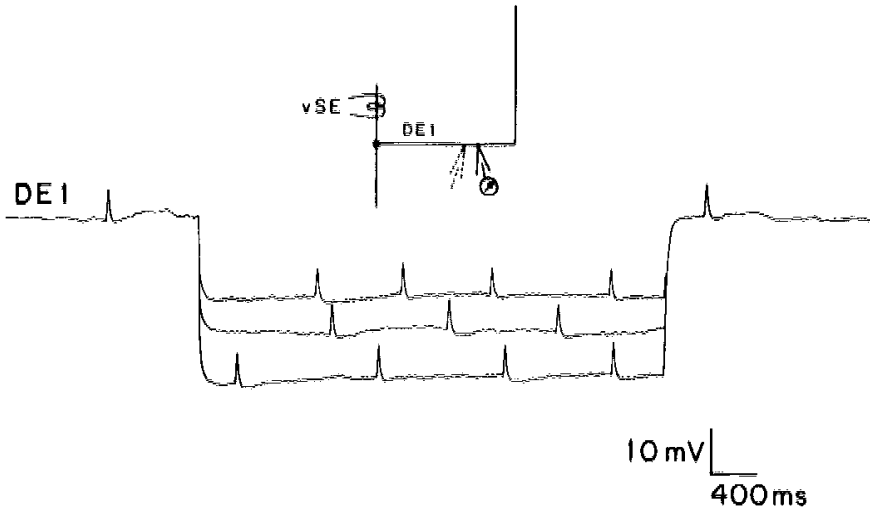

Figure 8. Suction electrode stimulation does not evoke all-or-none action potentials even at strongly hyperpolarized membrane potentials. Two microelectrodes were placed in the same DE1 motorncuron. One microelectrode (trace not shown) was used to inject hyperpolarizing current pulses of 3 different amplitudes into the commissure. The other microelectrode (trace shown) recorded the actual voltage to which the motorneuron was hyperpolarized below the resting potential (approx. $-55,-63$, and $-72 \mathrm{mV}$ ). Strong direct suction electrode evoked responses (manually triggered) were elicited before, during, and after the hyperpolarizing pulses. Resting potential, $-38 \mathrm{mV}$.

parable initial transient response, but if the presynaptic current pulse is sufficiently strong, the postsynaptic hyperpolarization may be followed by a depolarizing rebound that is graded with stimulus strength.

\section{Tonic release of neurotransmitter}

Hyperpolarization of excitatory motorneurons results in hyperpolarization of postsynaptic cells (Figs. 9, $g-j ; 11 e$ ) Hyperpolarization of inhibitory motorneurons results in depolarization of postsynaptic cells (Figs. 10B, 12d). A plausible explanation for these results is that commissural motorneurons release neurotransmitter tonically at rest and this hyperpolarization decreases release. Tonic release is a graded phenomenon: increasing hyperpolarizing current reduces neurotransmitter release from both excitatory and inhibitory motorneurons onto muscle in graded fashion (Figs. 9, 10; also see Figs. 13, 14). Tonic release has been observed for motorneuron-muscle interactions (Figs. $9,10)$ and for motorneuron-motorneuron interactions (Figs. 11, 12).

The depolarizing or hyperpolarizing plateau phase of postsynaptic responses can be maintained with little or no repolarization back to the resting potential even for current pulses of long duration (Fig. 15). Thus, commissural motorneurons are capable of releasing neurotransmitter tonically for extended periods of time without significant fatigue.

\section{Discussion}

While commissural motorneurons are capable of conducting signals passively over long distances (Davis and Stretton, 1989), they are also able to generate active signals. The presence of voltage-sensitive channels in commissural membranes is indicated by graded anodic/cathodic offset responses and oscillatory activity. All-or-none action potentials, however, could not be evoked, nor were they observed to occur spontaneously. Synaptic transmission is mediated without spikes and is therefore graded; in addition, transmitter release occurs tonically at normal resting potentials. 


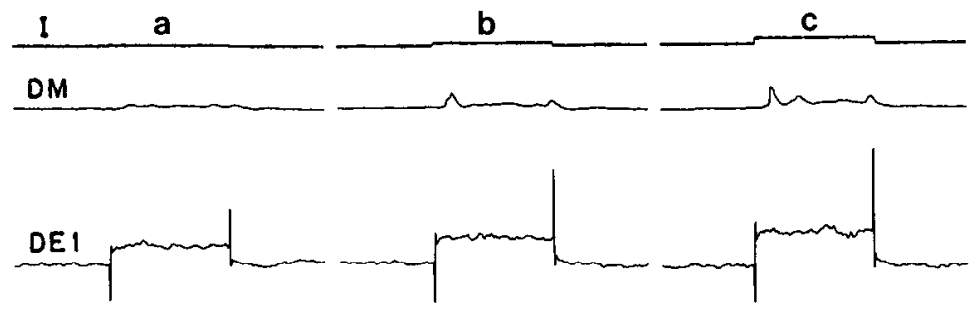

Figure 9. Graded synaptic transmission and tonic neurotransmitter release between an excitatory motorneuron and postsynaptic muscle. Injection of increasing depolarizing current $(a-f)$ into a DE1 motorneuron $(D E 1)$ results in a graded increase in the amplitude of depolarizing dorsal muscle $(D M)$ responses ( $I$, current monitor). Injection of increasing hyperpolarizing current $(g-$ $j$ ) into a DE1 motorneuron results in a graded increase in the amplitude of hyperpolarizing dorsal muscle responses.
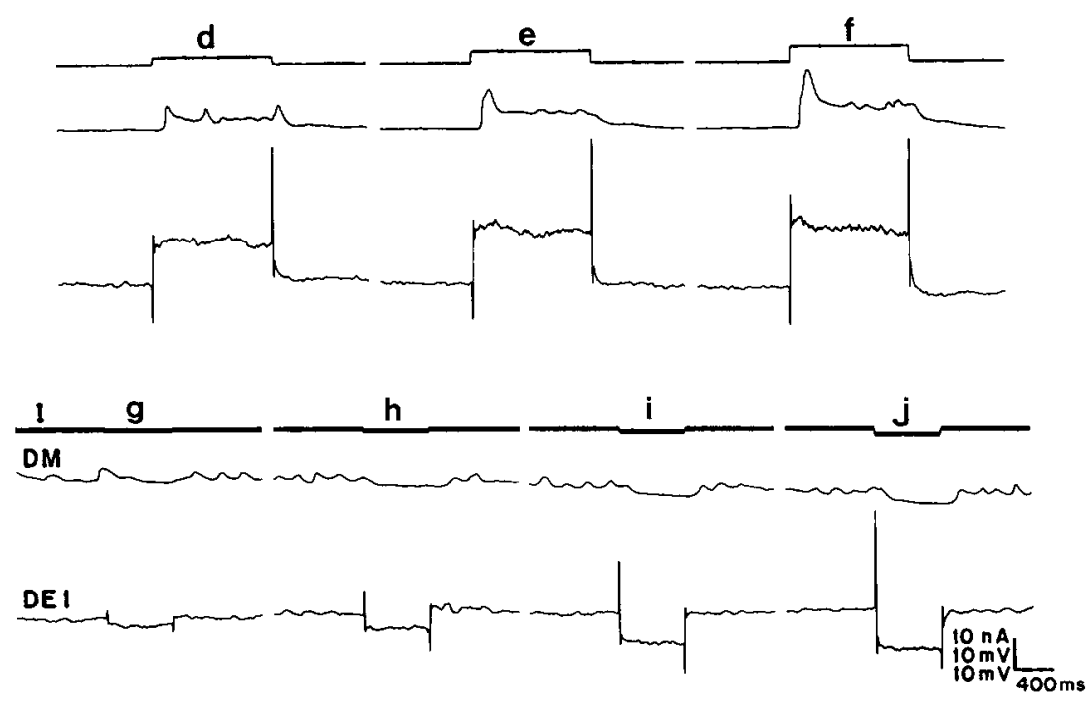

Intracellularly recorded commissural signals: anode- and cathode-break responses

At first glance it seems incongruous that active signals like anode- and cathode-break responses can be elicited in cells that are characterized by relatively linear $I-V$ plots (see Davis and Stretton, 1989). However, such active signals are elicited only with abrupt changes in current density such as occur at current pulse onsets or offsets. The small onset transient occasionally seen at the beginning of strong intracellularly injected current pulses is another example of the occurrence of active signals in an otherwise passively conducting system; its sensitivity to $\mathrm{Co}^{2+}$ suggests that it may be the result of activation of the same voltage-sensitive channels as those underlying anode-break re- sponses (see Fig. 9 in Davis and Stretton, 1989). Similar graded onset and offset transients have been reported in other nonspiking cells (Bush and Roberts, 1968; Graubard, 1978; Blight and Llinás, 1980; Oertel and Stuart, 1981). In many excitable cells of other animals, anode-break stimulation produces all-ornone action potentials. Under the conditions of these experiments, Ascaris motorneurons are not capable of generating all-or-none action potentials. Inactivation of channels due to damage-induced depolarization does not account for this since no response is elicited at membrane potentials strongly hyperpolarized with respect to the resting potential.

It is possible that the voltage-sensitive channels underlying the rising phase of the anode-break response are also responsible for neurotransmitter release, since both phenomena (1) rely on
Figure 10. Graded synaptic transmission and tonic neurotransmitter release between an inhibitory motorneuron and postsynaptic muscle. $A$, Injection of increasing depolarizing current into a VI motorneuron $(V I)$ results in a graded increase in the amplitude of hyperpolarizing ventral muscle $(V M)$ responses ( $I$, current monitor). $B$, Injection of increasing hyperpolarizing current into a VI motorneuron results in a graded increase in the amplitude of depolarizing ventral muscle responses.

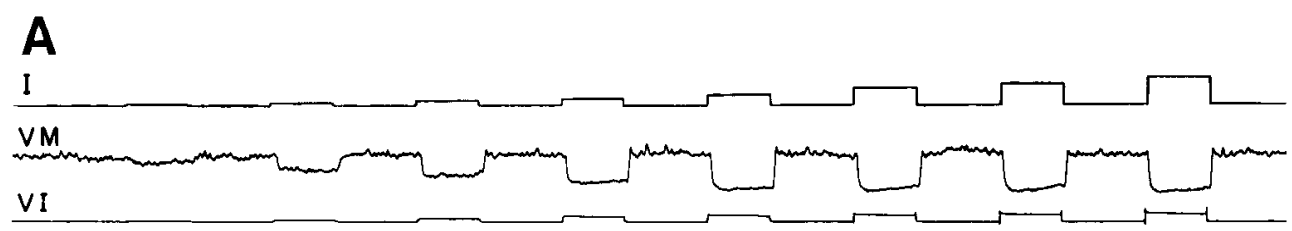

B

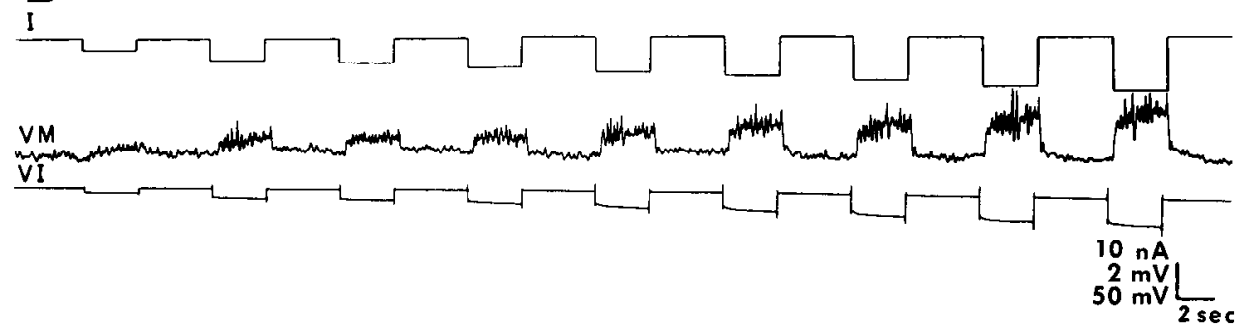



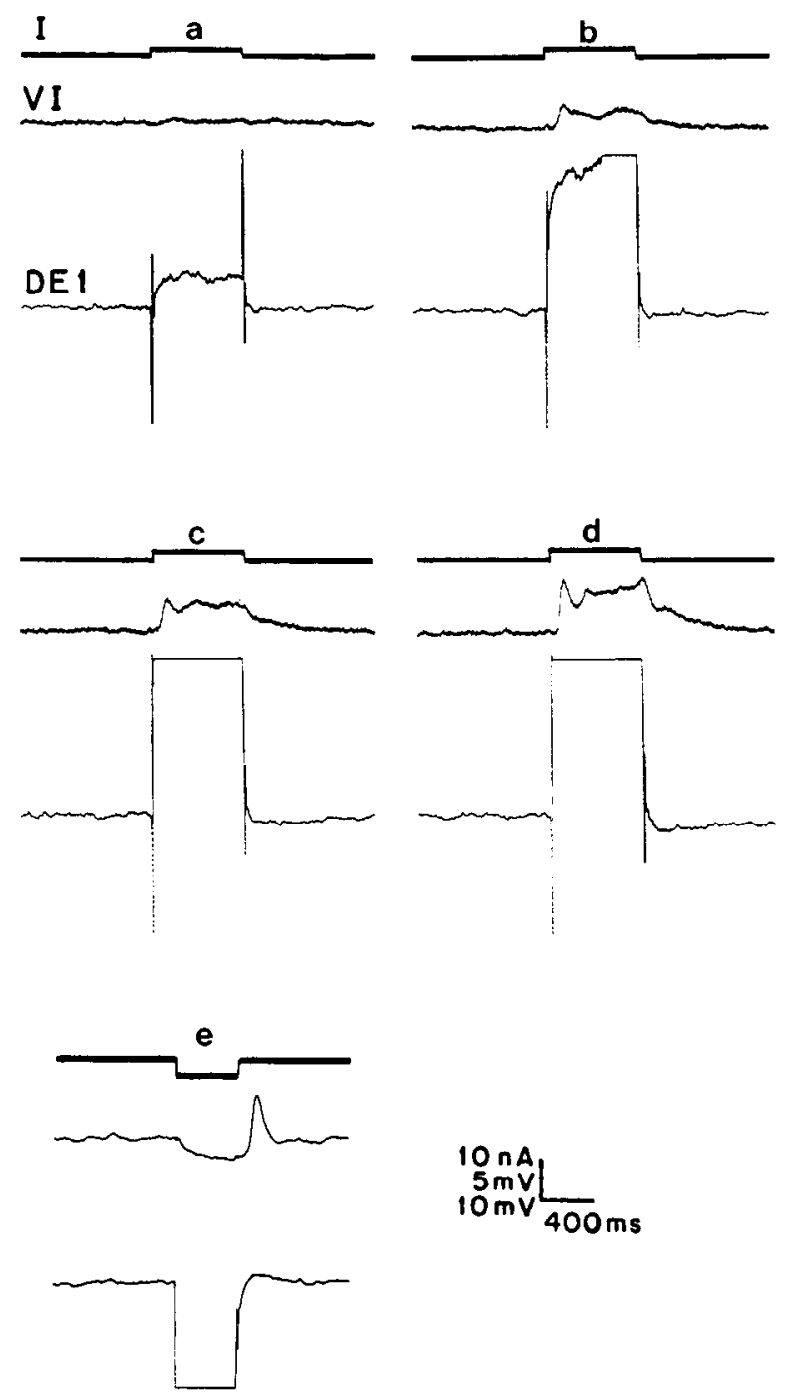

Figure 11. Graded synaptic transmission and tonic neurotransmitter release between an excitatory motorneuron and a postsynaptic inhibitory motorneuron. Injection of increasing depolarizing current $(a-d)$ into a DE1 motorneuron results in a graded increase in the amplitude of depolarizing VI motorneuron responses (I, current monitor). Injection of hyperpolarizing current $(e)$ into the DE 1 motorneuron results in a hyperpolarizing VI motorneuron response, suggesting tonic release of neurotransmitter (DE1 bridge circuitry unbalanced).

channels that have voltage sensitivities that activate below and appear to overlap the motorneuron resting potential, (2) are graded in their response to the potential of the membrane, and (3) are $\mathrm{Co}^{2+}$-sensitive, suggesting a possible $\mathrm{Ca}^{2+}$ dependence. If the putative $\mathrm{Ca}^{2+}$ channels underlying the anode-break response and neurotransmitter release are the same, then they are distributed not only over the synaptic output process of the motorneuron but also (to some extent) over other regions of the neuron since anode-break responses can be recorded in the commissure after it has been severed from its synaptic output process. As has been shown to be the case in other preparations (see Katz and Miledi, 1969; Baker et al., 1971; Edgington and Stuart, 1979), the density of $\mathrm{Ca}^{2+}$ channels might be higher in synaptic than in nonsynaptic regions.

The mechanism by which the anode-break response repolarizes to the resting potential is unknown. The relative linearity of $I-V$ plots (Davis and Stretton, 1989) suggests that a voltage-

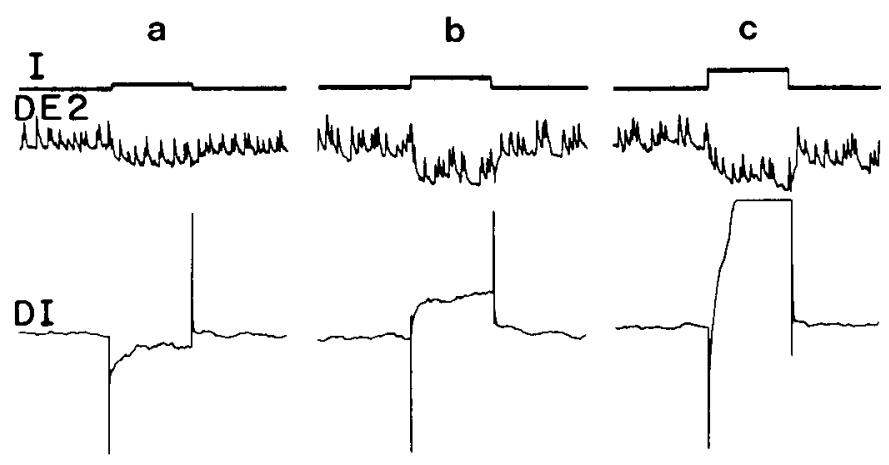

d
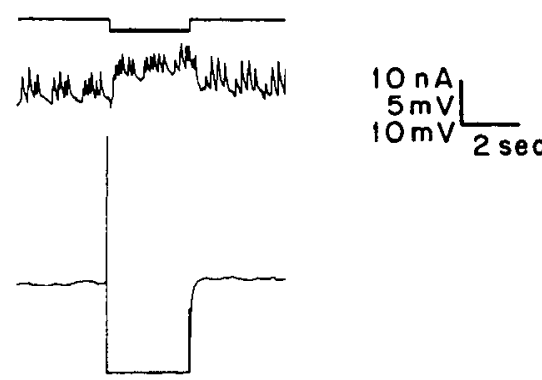

Figure 12. Graded synaptic transmission and tonic neurotransmitter release between an inhibitory motorneuron and a postsynaptic excitatory motorneuron. Injection of increasing depolarizing current $(a-c)$ into a DI motorneuron results in a graded increase in the amplitude of hyperpolarizing DE2 motorneuron responses ( $I$, current monitor). Injection of hyperpolarizing current $(d)$ into the DI motorneuron results in a depolarizing DE2 motorneuron response, suggesting tonic release of neurotransmitter. (DI bridge circuitry slightly overbalanced in $a$ and out of balance in $c$ and $d$ ).

sensitive delayed $\mathrm{K}^{+}$rectifier $\left(I_{K(\nu)}\right)$ is not participating to any significant degree in repolarization of the anode-break response. In the nonspiking crab stretch receptor (Blight and Llinás, 1980), a similar situation is encountered. The $I-V$ plot is linear, suggesting the absence of strong rectifying currents, yet the repolarization of a depolarizing onset transient must be explained. Blight and Llinás have hypothesized that an inactivating, voltage-sensitive potassium current could account for the repolarization of the transient and the lack of rectification in the $I-V$ plot.

In Ascaris, it is possible that the falling phase is purely passive. However, the existence of cathode-break responses suggests that active channels are involved. The cathode-break response in Ascaris motorneurons is similar to a voltage-sensitive hyperpolarizing response that Byerly and Masuda (1979) have studied in Ascaris pharyngeal muscle. Both responses can be evoked at the offset of depolarizations, and both reach their most negative extent approximately -5 to $-10 \mathrm{mV}$ below the resting potential (pharyngeal muscle resting potential, -35 to $-40 \mathrm{mV}$ ). Using voltage-clamp analysis, Byerly and Masuda have demonstrated that the current underlying the pharyngeal response is an inactivating voltage-sensitive potassium current. Hyperpolarization activates the negative-going current when the membrane sits at potentials depolarized with respect to the resting potential: the conductance turns on at $-15 \mathrm{mV}$ and saturates at potentials below $-30 \mathrm{mV}$.

Yet another possibility is that a $\mathrm{Ca}^{2+}$-activated potassium channel exists in these cells. Clearly, further experiments are required to resolve this issue. While active transient responses 
Figure 13. Graph of the relationship between the amount of current injected into an excitatory motorneuron (DE1) and the resulting potential change in plateau responses of the postsynaptic musclc (DM).

of the magnitude we have described for our elicited responses probably are not used biologically, the role of the conductances underlying them remains to be determined.

\section{Intracellularly recorded commissural signals: oscillatory activity}

The DI and VI commissural motorneurons are capable of producing rhythmic oscillatory activity. Oscillatory motorneurons in Ascaris hold much in common with the oscillator cells seen in other systems ( $c f$. Mendelson, 1971; Pearson and Fourtner, 1975; Heitler and Pearson, 1980). For example, they share the following features with oscillator cells in the lobster ventilatory rhythm (Mendelson, 1971): (1) low resting potentials (approximately $-36 \mathrm{mV}$ in the lobster), (2) periods of quiescence and spontaneous oscillatory activity, (3) depolarization-induced initiation of oscillations in quiet cells, (4) phase shifting with the appropriate current pulses, (5) gradedness in oscillation amplitude, (6) graded synaptic transmission, and (7) tonic neurotransmitter release.

As regards the nature of the oscillatory signal itself, there are many similarities between Ascaris oscillatory potentials and the repetitive signals described in the crab cardiac ganglion (Berlind, 1982; Benson and Cooke, 1984). The voltage-sensitive signals in the crab, known as driver potentials, have shapes, durations, and amplitudes similar to the signals we record in Ascaris; as in Ascaris, they can show variability in the waveform of the signal and gradedness in amplitude. In the crab, Podophthalmus, the cardiac neurons can be either quiescent or show a spontaneous rhythm of driver potentials (Berlind, 1982). In quiescent preparations, as in Ascaris, these signals can start up spontaneously or they can be evoked with depolarizing current pulses. Driver potentials can be suppressed with hyperpolarizing current pulses. It is thought that driver potentials may play a role in the generation of the rhythmic output by the cardiac ganglia (Berlind, 1982; Benson and Cooke, 1984).

In Ascaris, the oscillatory frequency is somewhat faster than the normal behavioral frequency. It is not yet clear whether this implies that there is no direct causative relationship between the two or whether the differences in frequency are due to possible physiological differences between the in vivo and in vitro conditions.

\section{Absence of all-or-none spikes}

The graded nature of signals in commissural motorneurons is paralleled by the graded nature of evoked responses in Ascaris muscle. Weak stimulation of muscle cells yields graded slow potentials. If the stimulus intensity is increased, graded muscle "spikes" can be elicited on top of the slow potentials. These responses, like those of commissural motorneurons, give no evidence of the classic $\mathrm{Na}^{+} / \mathrm{K}^{+}$action potential.

The nonspiking cells of nervous systems have been predominantly either sensory cells or central, local circuit interneurons (Roberts and Bush, 1981; Siegler, 1985). The discovery of the nonspiking nature of Ascaris motorneurons is significant because it is the first description of a motornervous system where most, if not all, of the motorneurons function on a nonspiking basis. In addition, this is, to the best of our knowledge, the first indication that the broad category of "motorneurons" can be added to the growing list of sensory cells and interneurons that rely on graded (nonspiking) signals for information transfer.

\section{Graded synaptic transmission}

Ascaris motorneurons can produce graded changes in the postsynaptic membrane potential of muscle cells and other commissural motorneurons. Curves relating the strength and polarity of presynaptic current to postsynaptic potential changes are sigmoidal in shape. Sigmoidal shape characterizes the presynaptic voltage/postsynaptic voltage curves for both nonspiking cells (Burrows and Siegler, 1978; Graubard, 1978; Blight and Llinás, 1980) and TTX-blocked spiking cells (Katz and Miledi, 1967). As others have noted, this similarity in shape suggests that the mechanisms underlying transfer properties at chemical synapses may be basically the same regardless of whether the presynaptic cell spikes or not.

In some spiking and nonspiking cells, long presynaptic current pulses produce a peak postsynaptic response followed by a decline to a lower plateau potential that is maintained for the duration of the pulse (Katz and Miledi, 1966; Graubard, 1978; Blight and Llinás, 1980). Such a peak-plateau response is commonly observed in Ascaris synaptic interactions as well. Part of the peak response may directly reflect activation of voltagesensitive onset responses in the presynaptic cell. However, such 


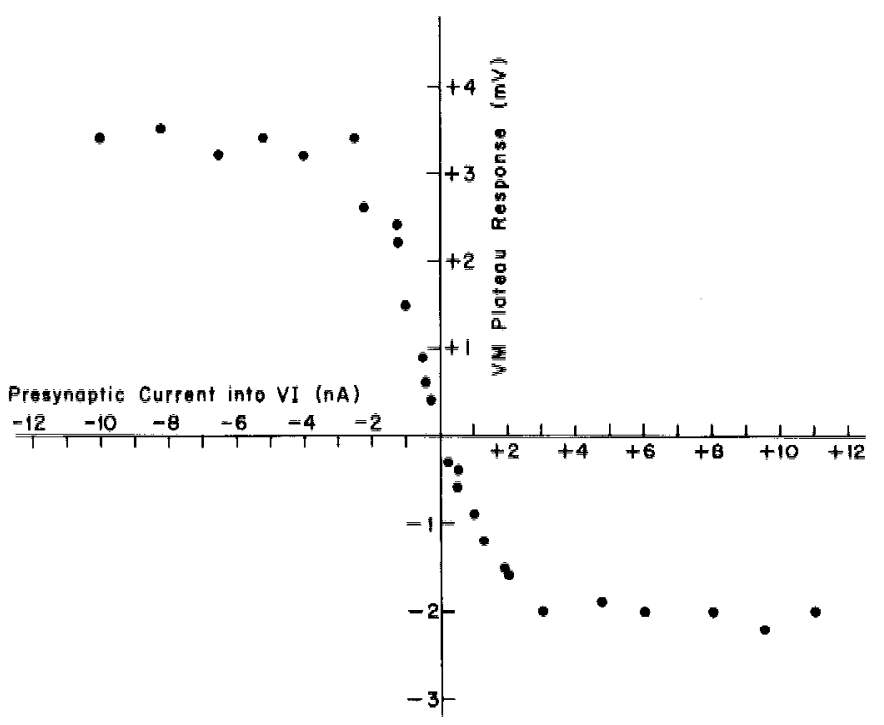

Figure 14. Graph of the relationship between the amount of current injected into an inhibitory motorneuron (VI) and the resulting potential change in plateau responses of the postsynaptic muscle (VM).

onset transients cannot fully account for it since a strong postsynaptic peak response is frequently obtained when there is little or no apparent presynaptic transient.

A number of lines of evidence indicate that these graded synaptic interactions involve chemical, and not electrical, synaptic transmission:

1. If $\mathrm{Co}^{2+}$ is substituted for $\mathrm{Ca}^{2+}$, neurotransmission in Ascaris commissural motorneurons is reversibly blocked (Fig. 3; cf. Fig. 9, Davis and Stretton, 1989).

2. Physiological and biochemical evidence indicate that $\mathrm{ACh}$ is an excitatory neurotransmitter in Ascaris motorneurons (del Castillo et al., 1963; Johnson and Stretton, 1985). Excitatory neuromuscular and motorneuron-motorneuron interactions are blocked by curare and other cholinergic blockers (Walrond and Stretton, 1985; J. D. Angstadt and A. O. W. Stretton, unpublished observations). In the case of inhibitory interactions, the reversal of sign (i.e., depolarizing currents producing hyperpolarizing responses and vice versa) is most easily explained by the release of an inhibitory neurotransmitter; preliminary physiological, biochemical, and immunological evidence suggests that GABA is an inhibitory neurotransmitter in Ascaris (del Castillo et al., 1963; Johnson and Stretton, 1987; S. J. Burden and A. O. W. Stretton, unpublished observations).

3 . An abundance of chemical synapses has been observed in the electron microscope for motorneuron-motorneuron and motorneuron-muscle neighbors (Stretton et al., 1978; J. E. Donmoyer, P. A. Desnoycrs, and A. O. W. Stretton, unpublished observations). These synapses contain the structures characteristic of chemical synapses, including conspicuous mitochondria, clusters of synaptic vesicles adjacent to the presynaptic membrane, and pre- and postsynaptic thickenings. In almost all cases where anatomical synapses have been scored, there is physiological evidence for the interaction, and conversely cell types lacking anatomically scored synapses between them also lack evidence of physiological interaction (Stretton et al., 1978; Walrond et al., 1985; R. E. Davis and A. O. W. Stretton, unpublished observations). Gap junctions have been electrophysiologically demonstrated and are commonly seen between muscle cells in
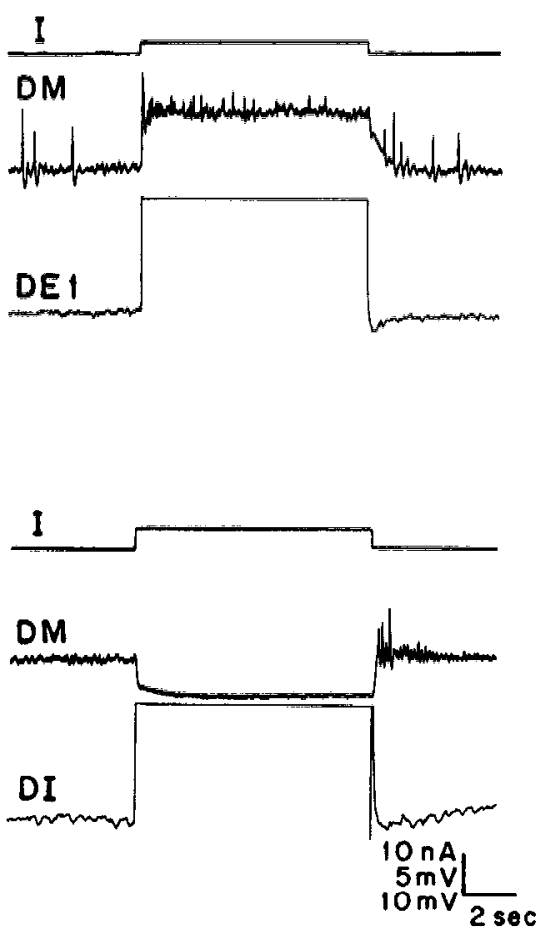

Figure 15. Motorneurons can release neurotransmitter for sustained periods of time without fatigue. Injection of $8.5 \mathrm{sec}$ depolarizing current pulses into motorneurons (top panel, DE 1; bottom panel, DI) result in postsynaptic dorsal muscle (DM) responses that persist for the duration of the pulse.

electron micrographs; however, they have not been observed between motorneurons and muscle, and are rare between motorneurons (Rosenbluth, 1965; J. E. Donmoyer, P. A. Desnoyers, and A. O. W. Stretton, unpublished observations).

The strongest evidence for chemical synaptic transmission would be demonstration of reversal potentials. Reversal potentials have been obtained for PSPs occurring in the motorneurons (R. E. Davis and A. O. W. Stretton, unpublished observations); however, it has not as yet been possible to obtain reversal potentials for the motorneuron-muscle synapses. Unfortunately, it is difficult to record muscle (or motorneurons) close to the site of these synapses. In addition, muscle cells are electrically coupled, have large surface areas, low input resistances (approximately $0.25 \mathrm{M} \Omega$ ), and complex geometries. These obstacles make it difficult to inject sufficient current into muscle cells so as to reveal the reversal potentials that would be expected to underlie chemically mediated PSPs.

\section{Tonic release of neurotransmitter}

The resting potential of Ascaris motorneurons lies approximately in the middle of the voltage-sensitive range of synaptic transmission. Thus, at rest there is tonic transmitter release that can be modulated up or down by changes in the membrane potential. del Castillo et al. (1963) observed that curare hyperpolarized and neostigmine depolarized Ascaris muscle cells. They therefore suggested that $\mathrm{ACh}$ was tonically released onto muscle, an interpretation consistent with our findings.

Evidence presented in the previous paper (Davis and Stretton, 1989) indicates that commissural motorneurons are not sustaining significant damage due to dissection or microelectrode penetration, and for the same reasons it is unlikely that the tonic release effect is a damage-induced phenomenon. In particular, 
following motorneuron penetration there is little or no change in the postsynaptic resting potential that would indicate that the rate of neurotransmitter release from the motorneuron has changed. In addition, after an hour or more of recording, the motorneuron resting potential remains stable and the tonic release effect is as robust as immediately following the penetration.

As is the case for the anode-break response, we do not know what prevents the tonically activated $\mathrm{Ca}^{2+}$ conductance at synapses from becoming all-or-none. $I-V$ plots have been carried out in the commissure but not in the synaptic region of the output process. Thus, we do not know the extent to which rectification occurs in this region. If the $\mathrm{Ca}^{2+}$ channels at the synapses are greater in density or have faster kinetics than those in the commissure, one would expect them to require a stronger counter-current. Since the synaptic conductances are continuously activated, it is unlikely that an inactivating $\mathrm{Ca}^{2+}$ conductance or an $I_{K(\mathrm{Ca})}$ provide an adequate explanation for the counter-current in Ascaris. It is not possible, however, to rule out a delicately balanced $I_{\mathrm{Ca}} / I_{K(\mathrm{Ca})}$ system that could, by negative feedback, maintain a stable resting potential level.

When the resting potential of a cell occurs within the rising phase of its transmitter release curve, it can communicate voltage differences of either sign to postsynaptic cells. In a system like Ascaris, in which oscillations in membrane potential may have to be accurately communicated to postsynaptic cells, this ability has obvious functional value. Cells with resting potentials in the steepest portion of their release curves also will be able to communicate small changes in presynaptic voltage with optimum effectiveness. The ability to signal even small deviations from the resting potential has been documented at high-sensitivity sensory synapses (e.g., photoreceptors, Shaw, 1972; Fain et al., 1977). It may also be necessary in systems like Ascaris, in which an initially strong input signal becomes smaller after decremental transmission over a long distance to the output synapses.

Tonic transmitter release in Ascaris may account for the muscle tonus thought to underlie the hydrostatic skeleton of $A$ scaris and perhaps other nematodes. Harris and Crofton (1957) demonstrated that Ascaris body wall muscles were capable of generating pressures high enough to account for the hydrostatic skeleton. Single worms showed wide and often rhythmical pressure variations. Tonic neurotransmitter release from motorneurons onto muscle may be responsible for this resting tonic contraction and coordinated changes in the tonic release from individual or sets of motorneurons could account for the rhythmical pressure variations. A role for tonic transmitter release in postural control has been suggested for other systems as well (Blight and Llinás, 1980).

Nonspiking cells that use graded synaptic transmission need not have resting potentials that normally lie within the release portion of their input-output curves. For example, the nonspiking EXl cell in the stomatogastric ganglion makes use of sustained graded synaptic transmission but only after it has been depolarized to the release threshold, which is approximately 13 $\mathrm{mV}$ above its normal resting potential of $-60 \mathrm{mV}$ (Graubard, 1978). However, a number of nonspiking cells have been found that may continuously release transmitter as part of their normal function (e.g., photoreceptors, local circuit interneurons). $A s$ caris motorneurons appear to fall in this latter category.

\section{References}

Angstadt, J. D. (1986) Anatomy and physiology of oscillatory neurons in the nematode Ascaris. Ph.D. dissertation, University of WisconsinMadison.

Baker, P. F., A. L. Hodgkin, and E. B. Ridgway (1971) Depolarization and calcium entry in squid giant axons. J. Physiol. (Lond.) 218: 709755.

Benson, J. A., and I. M. Cooke (1984) Driver potentials and the organization of rhythmic bursting in crustacea ganglia. Trends Neurosci. 7: 85-91.

Berlind, A. (1982) Spontaneous and repetitive driver potentials in crab cardiac ganglion neurons. J. Comp. Physiol. 149: 263-276.

Blight, A. R., and R. Llinás (1980) The non-impulsive stretch receptor complex of the crab; a study of depolarization-release coupling at a tonic sensorimotor synapse. Phil. Trans. R. Soc. London [Biol.] 290: 219-276

Burrows, M., and M. V.S. Siegler (1978) Graded synaptic transmission between local interneurones and motor neurones in the metathoracic ganglion of the locust. J. Physiol. (Lond.) 285: 231-255.

Bush, B. M. H., and A. Roberts (1968) Resistance reflexes from a crab muscle receptor without impulses. Nature 218: 1171-1173.

Byerly, L., and M. O. Masuda (1979) Voltage-clamp analysis of the potassium current that produces a negative-going action potential in Ascaris muscle. J. Physiol. (Lond.) 288: 263-284.

Davis, R. E., and A. O. W. Stretton (1989) Passive membrane properties of motorneurons and their role in long-distance signaling in the nematode Ascaris muscle. J. Neurosci. 9: 403-414.

del Castillo, J., W. C. de Mello, and T. Morales (1963) The physiological role of acetylcholine in the neuromuscular system of Ascaris lumbricoides. Arch. Int. Physiol. Biochem. 71: 741-757.

Edgington, D. R., and A. E. Stuart (1979) Calcium channels in the high resistivity axonal membrane of photoreceptors of the giant barnacle. J. Physiol. (Lond.) 294: 433-445.

Fain, G. L., A. M. Granda, and J. H. Maxwell (1977) Voltage signal of photoreceptors at visual threshold. Nature 265: 181-183.

Graubard, K. (1978) Synaptic transmission without action potentials: Input-output properties of a non-spiking presynaptic neuron. J. Neurophysiol. 41: 1014-1025.

Harris, J. E., and H. D. Crotton (1957) Structure and function in the nematodes: Internal pressure and cuticular structure in Ascaris. J. Exp. Biol. 34: 116-130.

Heitler, W. J., and K. G. Pearson (1980) Non-spiking interactions and local interneurons in the central pattern generator of the crayfish swimmeret system. Brain Res. 186: 206-211.

Hengstenberg, R. (1977) Spike responses of 'non-spiking' visual interneurone. Nature 270: 338-340.

Johnson, C. D., and A. O. W. Stretton (1985) Localization of cholineacetyltransferase within identified motoneurons of the nematode $A s$ caris. J. Neurosci. 5: 1984-1992.

Johnson, C. D., and A. O. W. Stretton (1987) GABA-immunoreactivity in inhibitory motor neurons of the nematode Ascaris. J. Neurosci. 7: 223-235.

Katz, B., and R. Miledi (1966) Input-output relation of a single synapse. Nature 212: 1242-1245.

Katz, B., and R. Miledi (1967) A study of synaptic transmission in the absence of nerve impulses. J. Physiol. (Lond.) 192: 407-436.

Katz, B., and R. Miledi (1969) Tetrodotoxin-resistant electrical activity in presynaptic terminals. J. Physiol. (Lond.) 203: 459-487.

Lowe, D. A., B. M. H. Bush, and S. H. Ripley (1978) Pharmacological evidence for "fast" sodium channels in nonspiking neurons. Nature 274: $289-290$.

Mendelson, M. (1971) Oscillator neurons in crustacean ganglia. Science 171: 1170-1173.

Oertel, D., and A. E. Stuart (1981) Transformation of signals by interneurons in the barnacle's visual pathway. J. Physiol. (Lond.) 311 : 127-146.

Pearson, K. G., and C. R. Fourtner (1975) Nonspiking interneurons in walking system of the cockroach. J. Neurophysiol. $38: 33-52$.

Roberts, A., and B. M. H. Bush, eds. (1981) Neurones without Impulses, Cambridge U. P., London.

Rosenbluth, J. (1965) Ultrastructure of somatic muscle cells in Ascaris 
lumbricoides. II. Intermuscular junctions, neuromuscular junctions and glycogen stores. J. Cell. Biol. 26: 579-591.

Shaw, S. R. (1972) Decremental conduction of the visual signal in barnacle lateral eye. J. Physiol. (Lond.) 220: 145-175.

Siegler, M. V. S. (1985) Nonspiking interneurons and motor control in insects. Adv. Insect Physiol. 18: 249-304.

Stretton, A. O. W., R. M. Fishpool, E. Southgate, J. E. Donmoyer, J. P. Walrond, J. E. R. Moses, and I. S. Kass (1978) Structure and physiological activity of the motorneurons of the nematode Ascaris. Proc. Natl. Acad. Sci. USA 75: 3493-3497.
Walrond, J. P., and A. O. W. Stretton (1985) Reciprocal inhibition in the motornervous system in the nematode Ascaris: Direct control of ventral inhibitory motorneurons by dorsal excitatory motorneurons. J. Neurosci. 5: 9-15.

Walrond, J. P., I. S. Kass, A. O. W. Stretton, and J. E. Donmoyer (1985) Identification of excitatory and inhibitory motorneurons in the nematode Ascaris by electrophysiological methods. J. Neurosci. 5: $1-8$. 